\title{
Caries experience and oral health related quality of life in a cohort of Ugandan HIV-1 exposed uninfected children compared with a matched cohort of HIV unexposed uninfected children
}

\author{
Nancy Birungi $i^{*}$, Lars T. Fadnes ${ }^{1,2}$, Ingunn M. S. Engebretsen ${ }^{3}$, Stein Atle Lie ${ }^{1}$, James K. Tumwine ${ }^{4}$,
} Anne Nordrehaug Åstrøm ${ }^{1}$ and for the ANRS 12174 and 12341 study groups

\begin{abstract}
Background: Very few studies consider the oral health status and quality of life in HIV-1 exposed uninfected (HEU) children. The aim of this study was to estimate the prevalence of caries in primary teeth and its oral health related quality of life impacts in HEU children compared to HIV-unexposed-uninfected (HUU) children, whilst adjusting for confounding covariates.

Methods: This study uses data from the Ugandan site of the ANRS 121741 PROMISE- PEP trial (ClinicalTrials.gov, number NCT00640263) conducted in 2009-2013 that recruited mothers with HIV-1 and their uninfected children. Of 244 HEU-children-caretaker pairs available at the end of the one-year trial, 166 were re-enrolled in the ANRS 12341 PROMISE-PEP M\&S study at 5-7 years and 164 were included in this study. These were age and sex-matched with 181 HUU children-caretaker comparators. Caries experience was recorded using World Health Organization's Decayed, Missed and Filled teeth (dmft/DMFT) indices. The Early Childhood Oral health Impact Scale (ECOHIS) was used for assessment of oral health related quality of life. Mixed effects logistic regression was conducted with dmft and ECOHIS scores as outcomes and HIV-1 exposure status as the main exposure.

Results: Forty-eight percent of HEU children and $60 \%$ of HUU had dmft $>0$. Corresponding figures for ECOHIS $>0$ were $12 \%$ of HEU and $22 \%$ of HUU. The crude analysis showed differences related to HIV-1 exposure in caries experience and oral health related quality of life. Mixed effect logistic regression analyses were not significant when adjusted for use of dental care and toothache. If caregivers' DMFT $>0$, the adjusted odds ratio for caries experience ( $\mathrm{dmft}>0$ ) was $1.6(95 \% \mathrm{Cl}: 1.0-2.8)$ while if $\mathrm{dmft}>0$ the adjusted odds ratio for quality of life impacts (ECOHIS>0) was 4.6 (95\% Cl: 2.0-10.6).

* Correspondence: Nancy.Birungi@uib.no

'Department of Clinical Dentistry, University of Bergen, P. O Box 7800 5020, Bergen, Norway

Full list of author information is available at the end of the article

C C The Author(s). 2020 Open Access This article is licensed under a Creative Commons Attribution 4.0 International License, which permits use, sharing, adaptation, distribution and reproduction in any medium or format, as long as you give appropriate credit to the original author(s) and the source, provide a link to the Creative Commons licence, and indicate if changes were made. The images or other third party material in this article are included in the article's Creative Commons licence, unless indicated otherwise in a credit line to the material. If material is not included in the article's Creative Commons licence and your intended use is not permitted by statutory regulation or exceeds the permitted use, you will need to obtain permission directly from the copyright holder. To view a copy of this licence, visit http://creativecommons.org/licenses/by/4.0/. The Creative Commons Public Domain Dedication waiver (http://creativecommons.org/publicdomain/zero/1.0/) applies to the data made available in this article, unless otherwise stated in a credit line to the data. 
(Continued from previous page)

Conclusion: The prevalence of untreated caries in primary teeth and quality of life impacts was high in this study population. HIV-1 exposed uninfected children were not more likely than HUU children to experience dental caries or have impaired oral health related quality of life. Given the global expansion of the HEU child population, the present findings indicating no adverse effect of pre- and post-natal HIV-1 exposure on caries in deciduous teeth are reassuring.

Keywords: Dental caries, Oral health, Quality of life, HIV unexposed infected, Children

\section{Background}

According to the Global Burden of Disease 2010 study, untreated caries in deciduous teeth or early childhood caries (ECC) ranks the 10th most prevalent condition affecting $9 \%$ of the global child population [1]. In high income countries dental caries is among the most common chronic infectious diseases in children with prevalence reported up to $50 \%$, whereas in low-income countries, caries in the primary dentition vary from 6 to $71 \%$ [2]. ECC leads to temporary pain and impacts on the quality of life of the child and family, including having financial and health implications [3].

Previous studies have confirmed that disadvantaged social status, exposure to adverse family environments and chronic diseases at early life stages constitute risk factors for developing dental caries in the primary dentition. Caretaker characteristics, such as poor feeding and rearing practices, parents' own caries situation and poor oral hygiene associate with dental caries in the primary dentition of their offspring [4-8]. The increased risk of dental caries, oral mucosal lesions and periodontal disease in populations with HIV-1 has been thoroughly documented [9-11]. Despite experience with antiretroviral therapy globally, caries prevalence of children with HIV-1 vary with prevalence rates of 85 and $20 \%$ in lowand high income countries, respectively [12, 13]. During recent decades, industrialized countries have reported on a decline in children's caries experience. In contrast, caries prevalence among children with HIV-1 have remained high in low- and high income countries [12, 14].

One important route of HIV-1 infection is transmission from mother to child accounting for $15-45 \%$ of transmissions without interventions. In sub Saharan Africa, implementation of antiretroviral therapy, ART, and improved counselling of postnatal risk factors such as infant feeding has dramatically reduced the number of infants being infected [15]. Even though the global plan to move towards elimination of new HIV-1 infections among children by 2015, was not achieved, tremendous success has been made in lowering the number of infants with HIV-1 [16]. Globally, between 2000 and 2014, the number of children acquiring HIV-1fell by $58 \%$ to 220,000 per year [16]. Concerted efforts coupled with increasing availability of effective prevention of mother-to-child treatment (PMTCT) interventions, make it possible to eliminate mother- to-child transmission in resource limited setting [17]. Since the coverage of effective interventions for mother to child transmission of HIV-1 is increasing faster than the antenatal HIV-1prevalence declines, a population of HIV exposed uninfected children (HEU) is emerging [18]. Every year above one million infants are born to women with HIV-1 worldwide, the majority of them in low income countries, and most of them will not become infected [19].

Studies from various settings have reported that HIV exposed, uninfected children (HEU) have poorer health outcomes than HIV unexposed uninfected children (HUU) [19-23]. Thus, HEU children seem to be disadvantaged with respect to birth outcomes such as preterm birth, linear growth, metabolism, adaptive and innate immune systems; and increased infectious morbidity [24]. In addition to direct exposure in utero and during delivery, mothers with HIV-1 can also affect the health situation of their children indirectly through poverty, caregiver insufficiency, unemployment, illness and bereavement [25]. Previous studies have shown that compared to HEU children, children with HIV infection have more negative impacts on their quality of life [26-28]. However, comparative studies for the quality of life of HEU children with HUU children are limited. Two studies from Uganda showed similar quality of life profiles among HUU and HEU children [27, 29]. Another study from Uganda found that infection/ exposure predicted low self-esteem and diminished positive outlook in the long term [28]. However, none of those studies investigated oral health related quality of life, specifically. Although HEU children are recognized to have specific health needs, very limited evidence is available regarding their survival, health, and oral health situation [14]. In addition, many cohort studies suffer from limited follow-up, lack of comparable control groups, inadequate consideration of confounding factors and inconsistent use of standardized and validated assessment tools [20]. 
This study compares caries in primary teeth and oral health related quality of life between a cohort of HEUchildren at age 5-7 years who received peri-exposure prophylaxis during the initial breastfeeding period after birth and an age- and sex- matched control group of HIV unexposed uninfected children. Thus, the aim was to estimate the prevalence of caries in deciduous teeth and its oral health related quality of life impacts in HIV exposed uninfected children as compared to HIV unexposed uninfected children whilst adjusting for confounding covariates.

\section{Method}

This study is based on data generated from the followup of the clinical HIV-1 peri-exposure prophylaxis with lopinavir-ritonavir ANRS12174 PROMISE- PEP trial (ClinicalTrials.gov, number NCT00640263) [30]. The ANRS12174 PROMISE- PEP trial is described in detail in a previous paper [30]. The trial conducted between 2009 and 2013, was a multi-center randomized trial including pregnant women with HIV-1, recruited at gestational age of $28-40$ weeks at antenatal clinics in four African sites; Ouagadougou, Burkina Faso; East London, South Africa; Mbale, Eastern Uganda; and Lusaka, Zambia. HIV-1 infected pregnant women intending to breastfeed were referred for further assessment of inclusion criteria and again with their HIV uninfected children for enrolment and randomization at day 7 postpartum. Infants were eligible for inclusion if they were: a singleton; breastfed at day seven by their mothers; had a negative HIV-1 DNA PCR and had received any PMTCT. Further inclusion criteria was mother aged 18 years or older, intending to continue breastfeeding, HIV1 infected, and not eligible for ART (either clinically or because CD4 count $>350$ cells $/ \mu \mathrm{L}$ at that time). All eligible mothers and infants followed the routine national mother-to-child transmission prophylaxis (PMTCT) with antepartum zidovudine, intrapartum nevirapine, zidovudine-lamivudine for mothers and nevirapine for infants for 7 days postpartum.

In Uganda, 278, seven-day old uninfected children born to HIV-1 infected women, were randomized to receive infant prophylaxis (either lamivudine, 3TC or boosted lopinavir-ritonavir, LPV/r daily) throughout the breastfeeding period from day 7 to 50 weeks. The primary outcome was mother to child HIV-1 transmission, diagnosed every 3 months with HIV-1 DNA PCR between 7 days and 50 weeks post-delivery. Infection rates, and clinical and biological severe adverse events did not differ between the two drug regimens suggesting that infant HIV-1 prophylaxis with either drug was not superior as both led to very low rates of HIV-1 postnatal transmission during 50 weeks of breastfeeding [30].
In 2017, 244 out of 278 mothers with HIV-1 infection and their uninfected children were eligible for reenrollment in the follow-up study; the PROMISE-PEP Mechanism Safety study (PROMISE-PEP M\&S Ritonavir ANRS12341). Of the 166 HEU children re-enrolled, 2 were excluded due to HIV-1 conversion. Thus, $68 \%$ of the eligible cohort of HEU children $(164 / 244)$ was followed up with $32 \%(n=112)$ missed due to attrition. A comparison group of 199 HUU children matched on age and sex, as well as their HIV uninfected mothers, were recruited from communities located in Mbale, Eastern Uganda; which was the site for the ANRS12174 PROMISE- PEP trial. Of the 199 HUU control children 19 were excluded due to a positive HIV-1 test result, leaving $181 \mathrm{HUU}$ children and their uninfected mothers enrolled. This study uses information from interviews and clinical oral examinations of 164 HIV-1 infected caregiver-HEU child pairs and 181 age-and sex-matched HUU children and their caregivers at the follow-up in 2017.

\section{Interviews with mothers of the HEU and HUU children at follow-up}

Trained interviewers performed face-to-face interviews with caregivers before the children underwent oral clinical examination using semi-structured interviews in the local language Lumasaba. The interview was constructed in English and translated into Lumasaba for use in the field. The schedule had been reviewed previously by project staff for semantic, experiental and conceptual equivalence of the source version. Sensitivity to culture and selection of appropriate words were considered [31]. Caregivers responded to questions about themselves and their children. Information was documented on case record forms (CRFs) and electronically with Capture software System.(Clinsight) and Epidata progaram www. epidata.dk for the clinical oral examinations.

Socio-demographic characteristics of caregivers were assessed in terms of level of education, type of income and marital status. Level of education was categorized into: did not finish primary school (1), end of primary school (2) higher education (3). Marital status was categorized: divorced (1), cohabiting/married (2), single (3), widowed (4) and recoded into single/divorced/widow (0) and married/cohabiting (1). Mother's behavioral characteristics were assessed in terms of tooth brushing frequency (Categories of the socio-demographic and behavioral covariates are shown in Table 1). Type of income was categorized into (1) no regular income (2) regular income. Child's characteristics were assessed in terms of dental care utilization, tooth brushing, ever toothache, and breastfeeding duration.

Child impact section, (CIS) of the early childhood oral health impact scale (ECOHIS) scale for child's oral 
Table 1 Socio-demographic- clinical and behavioral characteristics of mother and child by child's HIV-1 status at follow-up in the Ugandan part of the PROMISE PEP M\&S study

\begin{tabular}{|c|c|c|c|}
\hline & HIV- exposed uninfected $(n=164)$ & HIV- unexposed uninfected $(n=181)$ & $\begin{array}{l}\text { Total } \\
(n=345)\end{array}$ \\
\hline Child characteristics & $\%(n)$ & $\%(n)$ & $\%(n)$ \\
\hline \multicolumn{4}{|l|}{ Sex } \\
\hline Male & $51(84)$ & $50(89)$ & $51(175)$ \\
\hline Female & $49(80)$ & $50(92)$ & $49(170)$ \\
\hline \multicolumn{4}{|l|}{ Age } \\
\hline 5 year & $28(46)$ & $31(56)$ & $29(29)$ \\
\hline 6-7 year & $72(118)$ & $69(125)$ & $70(243)$ \\
\hline \multicolumn{4}{|l|}{ Ever taken to dental care } \\
\hline No & $94(155)$ & $86(156)$ & $90(31)$ \\
\hline Yes & $5(9)^{a}$ & $14(25)$ & $10(34)$ \\
\hline \multicolumn{4}{|l|}{ Toothbrush } \\
\hline Less once a day & $20(33)$ & $10(17)$ & $15(50)$ \\
\hline Once a day or more & $80(133)^{b}$ & $90(160)$ & $85(292)$ \\
\hline \multicolumn{4}{|l|}{ Ever toothache } \\
\hline No & $90(147)$ & 79 (143) & $84(291)$ \\
\hline Yes & $11(17)^{b}$ & $21(38)$ & $16(55)$ \\
\hline \multicolumn{4}{|l|}{ Breastfeeding duration } \\
\hline $0-6$ months & $31(51)$ & $3(5)$ & $16(56)$ \\
\hline 7-12 months & $65(106)$ & $13(24)$ & $38(130)$ \\
\hline$>12$ months & 4. $(7)^{b}$ & $84(149)$ & $46(156)$ \\
\hline \multicolumn{4}{|l|}{ Mother /caretaker characteristics } \\
\hline \multicolumn{4}{|l|}{ Age } \\
\hline $18-32 y r$ & $40(58)$ & $58(104)$ & $50(162)$ \\
\hline $33+$ & $60(90)^{b}$ & $42(76)$ & $50(166)$ \\
\hline \multicolumn{4}{|l|}{ Toothbrush } \\
\hline No & $59(97)$ & $44(78)$ & $52(176)$ \\
\hline Yes & $40(66)^{b}$ & $56(99)$ & $48(165)$ \\
\hline \multicolumn{4}{|l|}{ Maternal CD4 count at birth } \\
\hline$>500$ cells $/ \mathrm{mm} 3$ & $98(60)$ & - & \\
\hline$\leq 500$ cells $\mathrm{mm} 3$ & $66(40)$ & - & \\
\hline \multicolumn{4}{|l|}{ Marital status } \\
\hline Single/divorced, widow & $26(43)$ & $18(33)$ & $22(76)$ \\
\hline Married/cohabiting & $74(121)^{a}$ & $82(147)$ & $78(268)$ \\
\hline \multicolumn{4}{|l|}{ Type of income } \\
\hline No regular & $60(98)$ & $72(130)$ & $66(229)$ \\
\hline Regular & $40(65)^{a}$ & $28(51)$ & $34(116)$ \\
\hline \multicolumn{4}{|l|}{ Educational level } \\
\hline Primary school & $38(54)$ & $40(65)$ & $39(120)$ \\
\hline End of primary school & $20(28)$ & $22(37)$ & $21(65)$ \\
\hline Middle school/high school/college & $42(59)$ & $38(63)$ & $40(122)$ \\
\hline
\end{tabular}

${ }^{a} p<0.05 ; x^{2}$ test, ${ }^{b} p<0.001 ; x^{2}$ test 
health related quality of life was assessed using six of its original nine questions [32]. The ECOHIS scale has previously been tested for its psychometric properties in the context of Ugandan pre-school children [33]. Caregivers were asked "has your child: ever had tooth ache, ever had swollen/bleeding gums, ever cried because of pain in mouth, ever failed to sleep because of pain in mouth, ever refused to eat because of pain in mouth, and ever refused to play because of pain in mouth. Dummy variables $(0=$ no, $1=$ yes $)$ were summed into a count variable (range $0-6$ ) and dichotomized into $0=$ no child impacts, $1=$ at least one child impact. Family impact section, FIS, was assessed using the four original questions. "Because of child's dental problems, how often have you or the other parent: taken time off from work, been upset, felt guilty and had financial problems? Response categories were rated on a 5 point scale from $0=$ never to $4=$ almost daily. Each item was dichotomized and the dummy variables were summarized into a count variable (range $0-4)$. The sum score was dichotomized into $0=$ no family impacts and $1=$ at least one family impact. A total ECOHIS score was constructed by adding the Child impact- and Family impact scores.

\section{Clinical oral examination of children and mothers at follow up}

Two experienced and calibrated dental surgeons (NB and $\mathrm{MM}$ ) performed a full-mouth oral clinical examination among children and their caregivers. Duplicate examinations, for inter-rater and intra-rater reliability were performed with 26 children not part of the ANRS12341PROMISE- PEP trial cohort.

Dental caries was assessed on fully erupted primary/ permanent teeth in children and caregivers using the decayed, missing, and filled teeth indexes (dmft/ DMFT) in accordance with the World Health Organization (WHO) guidelines for field conditions [34]. A tooth was documented as decayed if it was visually cavitated with the aid of a dental mirror and periodontal probe (Michigan $\mathrm{O}$ probe), and recorded as missing when extracted due to caries, as confirmed by the caregiver. For analyses, dental caries was denoted in two ways: presence or absence of $\mathrm{dmft} /$ DMFT and the total number of decayed, missed and filled teeth in caregivers and their children with $\mathrm{dmft} /$ DMFT scored 0 and 1 for presence /absence of caries experience in children and caregivers. Number of erupted (fully emerged recorded as free occlusal /incisal surface) permanent teeth and number of primary teeth maintained were counted for children with mixed dentition. Since dental caries in the permanent dentition of children was very limited, DMFT was not calculated for children's permanent teeth.
Maternal and child HIV-1 status at baseline and follow-up HIV-1 status of the HEU children was assessed using the HIV-1 DNA polymerase chain reaction from dried bloodspots. Mothers and children in the comparison group were tested for their HIV-1 status using serial and parallel HIV rapid testing with Determine, Stat-Pak and Uni-Gold, three test algorithm as recommended by the Ugandan Ministry of Health [35].

\section{Anthropometric status for caregiver-child pairs at follow -up}

At the 5 year follow-up, anthropometric measurements for all the participants (caregivers - child pairs) in terms of weight and height were collected twice according to WHO guidelines (http://www.who.int/childgrowth/training/en/) using Seca- brand scales and Stadiometers to the nearest decimal place.

\section{Statistical analysis}

STATA 15 (College Station, Texas 77,845 USA) was used for data analysis. Chi-square tests for (categorical) variables and independent sample T-tests (continuous variables) were used to assess differences in baseline characteristics between HEU children lost to follow-up and those who retained in the cohort, and for the crude associations between HIV-1 exposure status, covariates and the outcome variables. The data presented had a clustered two-level hierarchical structure with individuals clustered as matched HEU/HUU pairs. Ignoring that observations in a cluster are correlated (that is intra cluster dependency) usually leads to an underestimation of the standard errors, too narrow confidence intervals and higher Type 1 error rates. As clustering in these data were considered quite limited, multiple variable analyses were conducted with ordinary logistic regression, OLR, providing population averaged estimates. Secondly, multilevel random intercept logistic models (RIM) were fitted using mixed effects logistic regression. The RIM model explicitly allows for clustering by including both intra- and inter cluster variation in the model. Behavioral and clinical covariates statistically significantly associated both with HIV-1 exposure status and the outcomes of dental caries and oral quality of life were included in the multilevel logistic regression models as potential confounding variables. The effect of clustering (HEU/HUU pairs) was assessed by calculating intra class correlation coefficients (ICC) expressing variations between clusters as a proportion of the total variance (within plus between cluster variance). ICC varies from 0 , which implies independent observations within cluster to 1 indicating no within cluster variation. Hence, high values of ICC implies dependency between observations within cluster. A likelihood -ratio test was calculated to test the null hypothesis that ICC equals 0 . 
Rejection of the null hypothesis implies that the multilevel model is preferable.

\section{Results}

Cohort participants (HEU children and their mothers with HIV) followed up $(n=164)$ did not differ significantly from those lost to attrition $(n=80)$ regarding socio-demographic- and behavioral characteristics at baseline, 7 days post-partum. More than $90 \%$ of the caregivers of HEU and HUU children included in the followup study were mothers. More than half (60\%) of HIV-1 infected mothers followed up presented with CD4 counts $>500$ cells $/ \mu \mathrm{L}$ (median 524, IQR 439-515) at baseline. The calibration exercise comparing $\mathrm{dmft}$ scores for each primary tooth within and between examiners revealed intra- and inter-rater reliability of median Kappa (interquartile range-IQR) of $0.6(0.5-0.7)$ and 0.7 $(0.5-0.8)$, respectively. The corresponding scores for the DMFT of caregivers were $0.7(0.5-0.9)$ and $0.6(0.4-0.8)$, respectively.

Table 1 depicts the distribution of maternal- and child's socio-demographic- and behavioral characteristics at follow-up according to child's HIV-1 exposure status. A total of $50 \%$ of the HUU children were females and $69 \%$ were $5-7$ years old (Table 1). Corresponding figures for HEU children were 49 and $72 \%$, respectively. HEU children were more likely to have older caretakers (33 years and above) than their uninfected counterparts (60\% versus $42 \% p<0.0001$ ), and more likely to have caretakers that were of single or divorced marital status (26\% versus $18 \% p<0.05)$. Regular income was more prevalent in the HEU group compared to the HUU group ( $40 \%$ versus $28 \% \mathrm{p}<0.05)$. Breastfeeding for more than 12 months was more common among HUU children than among their HEU counterparts (84\% versus $4 \%, p<0.001)$. Tooth brushing was most prevalent among HUU children and more prevalent among uninfected than among infected mothers.

As shown in Tables 2, 48\% of HEU children had at least one missed, filled or decayed primary tooth $(\mathrm{dmft}>$ $0)$ and at least one tooth with untreated caries $(\mathrm{dt}>0)$. The corresponding figure among HUU children was 60 and $57 \%,(p<0.001)$. The prevalence of permanent decayed teeth (DT) was 4 and 5 in HEU and HUU children, respectively. The prevalence of caries experience (DMFT $>0)$ in HIV-1 infected and uninfected mothers was 81 and $71 \%, p<0.001$. Corresponding figures for mean DMFT were 4.6 and 2.8. The mean number of erupted permanent teeth in HEU and HUU children was respectively, 4.2 and $6.3(p<0.001)$. Corresponding figures regarding remained primary teeth were 17.4 and 14.7 ( $\mathrm{p}<0.001)$. Children's anthropometric measures in terms of WAZ, HAZ and BAZ did not differ according to children's HIV-1exposure status.

Table 3 depicts responses to the ECOHIS inventory according to each separate item in the total study group as well as among HEU and HUU children. As shown, the prevalence of child impacts (CIS) amounted to 12 and $22 \%(\mathrm{p}<0.001)$ in HEU and HUU children, respectively. Corresponding figures for FIS and total ECOHIS scores were $6 \%$ versus 8 and $12 \%$ versus $22 \%$. The prevalence of each separate CIS and FIS impact was consistently higher among HUU children than among their HEU counterparts. The internal consistency reliability in

Table 2 Dental caries experience and anthropometric status of mother and child by child's HIV-1 exposure status at follow-up in the Ugandan part of the PROMISE PEP M\&S study

\begin{tabular}{|c|c|c|}
\hline & HIV- exposed uninfected $(n=164) \%(n)$ & HIV- unexposed uninfected $(n=181) \%(n)$ \\
\hline \multicolumn{3}{|l|}{ Children } \\
\hline $\mathrm{dmft}^{\mathrm{c}}>0 \%(\mathrm{n})$ & $48(79)^{a}$ & $60(109)$ \\
\hline Mean $\mathrm{dmft}^{\mathrm{c}}(\mathrm{sd})$ & $2.0(3.1)$ & $2.1(2.7)$ \\
\hline$d t>0 \%(n)$ & $48(77)$ & $57(104)$ \\
\hline Mean dt $(s d)^{d}$ & $1.9(2.8)$ & $1.9(2.5)$ \\
\hline DT $>0 \%(n)$ & $4(7)$ & $5(10)$ \\
\hline No of erupted permanent teeth mean (sd) & $4.2(3.8)^{\mathrm{b}}$ & $6.3(5.8)$ \\
\hline No of maintained primary teeth mean (sd) & $17.4(7.2)^{b}$ & $14.7(7.5)$ \\
\hline WAZ $Z^{\mathrm{e}}$ weight for age $(s d)^{d}$ & $-0.71(1.1)$ & $-0.70(1.2)$ \\
\hline HAZ $Z^{f}$ height for age $(s d)^{d}$ & $-0.72(2.0)$ & $-0.51(1.8)$ \\
\hline WHZ ${ }^{g}$ weight for height $(s d)^{d}$ & $-0.30(1.7)$ & $-0.53(1.1)$ \\
\hline \multicolumn{3}{|l|}{ Mothers } \\
\hline $\mathrm{DMFT}^{\mathrm{C}}>0 \%(\mathrm{n})$ & $81(133)^{b}$ & $71(129)$ \\
\hline Mean $\mathrm{DMFT}^{\mathrm{c}}(\mathrm{sd})^{\mathrm{d}}$ & $4.6(5.3)^{b}$ & $2.8(3.2)$ \\
\hline
\end{tabular}

${ }_{\text {a }} p<0.05 ; \chi^{2}$ test $_{1}^{\mathrm{b}} p<0.001 \mathrm{x}^{2}$ test, ${ }_{1}^{\mathrm{c}} \mathrm{dmft} / \mathrm{DMFT}$ - Decayed, missing, filled teeth, ${ }^{\mathrm{d}}$ standard deviation ${ }^{\mathrm{e}}$ weight for age $\mathrm{z}$ score, ${ }^{\mathrm{f}}$ height for age $\mathrm{z}$ score, ${ }^{\mathrm{g}}$ weight for height $z$ score 
Table 3 Frequency distribution and Cronbach's alpha of child impact-, family impact-, and total scores by HIV-1 status at follow -up in the Ugandan part of PROMISE PEP M\&S study

\begin{tabular}{|c|c|c|c|}
\hline Items & $\begin{array}{l}\text { Total } \\
\%(n)\end{array}$ & $\begin{array}{l}\text { HIV-1 exposed uninfected } \\
\%(n)\end{array}$ & $\begin{array}{l}\text { HIV- unexposed uninfected } \\
\%(n)\end{array}$ \\
\hline \multicolumn{4}{|l|}{ Child impact- Has child: } \\
\hline Ever had toothache & $16(55)$ & $10(17)^{a}$ & $21(38)$ \\
\hline Ever had swollen/bleeding gums & $10(35)$ & $7(11)^{\mathrm{a}}$ & $13(24)$ \\
\hline Ever cried because of pain in mouth & $12(41)$ & $9(15)$ & $14(26)$ \\
\hline Ever failed to sleep because of pain in mouth & $10(33)$ & $7(12)$ & $11(21)$ \\
\hline Ever refused to eat because of pain in mouth & $10(36)$ & $8(13)$ & $13(23)$ \\
\hline Ever refused to play & $8(29)$ & $5(8)^{\mathrm{a}}$ & $1(21)$ \\
\hline \multicolumn{4}{|l|}{ Family impact } \\
\hline \multicolumn{4}{|c|}{ How often have you or another family member because of problems with child's mouth and teeth----- } \\
\hline Taken time off work & $6(20)$ & $7(11)$ & 5. (9) \\
\hline Been upset & $4(13)$ & $2(4)$ & $5(9)$ \\
\hline Felt guilty & $5(16)$ & $3(5)$ & $6(11)$ \\
\hline \multirow[t]{2}{*}{ Had financial difficulties } & $3(9)$ & $2(4)$ & $3(5)$ \\
\hline & \multicolumn{3}{|c|}{ Cronbach's alpha } \\
\hline Child impact score $(\mathrm{CIS})>0$ & 0.95 & $12(19)^{a}$ & $22(40)$ \\
\hline Family impact score (FIS) $>0$ & 0.86 & $6(10)$ & $8(14)$ \\
\hline ECOHIS total score $>0$ & 0.93 & $12(20)^{a}$ & $22(38)$ \\
\hline
\end{tabular}

${ }^{a} p<0.05 ; x^{2}$ test

terms of Cronbach's alpha was 0.95 (CIS score), 0.86 (FIS score) and 0.93 (Total ECOHIS score).

Table 4 depicts odds ratios (OR) estimates and 95\% confidence intervals for dental caries experience (dmft> 0 ) in primary teeth by HIV-1 exposure status and covariates in unadjusted and adjusted analyses. No crude association occurred between caries experience and socio-demographic factors except for type of income. Caries experience differed statistically significantly between exposed and unexposed children with 48 and $60 \%$ having $\mathrm{dmft}>0$, respectively. Child's toothache, child's experience with dental care and caretakers' caries experience associated statistically significantly with children's caries experience as well as their HIV-1 exposure status and were included as possible confounding factors in the multiple variable logistic regression models. Mixed model logistic regression revealed that HEU children were not more likely than HUU children to have dental caries (OR 0.7, 95\% CI: 0.4-1.2). Cluster specific ORs based on mixed model logistic regression revealed the following significant covariates; ever taken to dental care (OR 3.1, 95\% CI: 1.1-8.9), ever experienced toothache (OR 7.8, 95\% CI: 2.9-20.7), mothers' tooth brushing (OR 1.6, 95\% CI: 1.0-2.6), mothers' DMFT (OR 1.6, 95\% CI: 1.0-2.8) and regular income (OR 1.7, 95\% CI: 1.0-2.8). No two-way interaction occurred between HIV-1 exposure status and other covariates (Supplementary Table 1, Additional file 1).
Table 5 depicts the unadjusted and adjusted associations from cross tabulation, ordinary logistic regression analyses and mixed model logistic regression regarding the total ECOHIS scores by HIV-1 status and potentially confounding covariates. ECOHIS scores differed statistically significantly between exposed and unexposed children with respectively, 12 and 22\% having ECOHIS score $>0$. Adjusted cluster specific ORs based on mixed model analyses was 4.6 (95\% CI: 2.0-10.6) regarding child's caries experience and 5.0 (95\% CI 1.8-13.6) regarding ever been taken to dental care. HIV-1 exposure status and breastfeeding duration did not remain statistically significantly associated with ECOHIS scores in adjusted multiple variable regression models. There were no significant interactions observed (See supplementary Table 2, Additional file 1). The intra class correlation (95\% CI) estimated was limited amounting to ICC (95\% CI: 0.2 , (0.02-0.73) implying that $20 \%$ of the variance in ECOHIS scores explained by covariates was between clusters and $80 \%$ within clusters.

\section{Discussion}

This study is among the first to report on caries in primary teeth and its oral health related quality of life impacts of 5-7 year-old HEU children as compared to their HUU counterparts living in a similar non-occidental cultural setting. At the time of the present study, no other published study comparing both clinical- and subjective 
Table 4 Children's dental caries experience (dmft>0) by HIV.1 exposure status socio-demographic- clinical and behavioral characteristics. Unadjusted and adjusted ordinary logistic regression and mixed model logistic regression

\begin{tabular}{|c|c|c|c|c|}
\hline Child characteristics & Cross tabulation & $\begin{array}{l}\mathrm{OLS} \\
\mathrm{dmft}>0\end{array}$ & $\begin{array}{l}\text { Adjusted OLS } \\
\mathrm{dmft}>0\end{array}$ & $\begin{array}{l}\text { Adjusted RIM }{ }^{d} \\
d m f t>0\end{array}$ \\
\hline HIV-1Status & $\%(n)$ & OR $(95 \% \mathrm{Cl})^{\mathrm{e}}$ & OR $(95 \% \mathrm{Cl})^{\mathrm{e}}$ & OR $(95 \% \mathrm{Cl})^{\mathrm{e}}$ \\
\hline HIV-1 unexposeed uninfected & $60(109)$ & 1 & 1 & 1 \\
\hline HIV exposed uninfected & $48(79)^{a}$ & $0.6(0.4-0.9)^{f}$ & $0.8(0.4-1.2)$ & $0.7(0.4-1.2)$ \\
\hline \multicolumn{5}{|l|}{ Sex } \\
\hline Male & $58(102)$ & 1 & & \\
\hline Female & $51(87)$ & $0.8(0.5-1.2)$ & & \\
\hline \multicolumn{5}{|l|}{ Age } \\
\hline 5 year & $55(56)$ & 1 & & \\
\hline 6-7year & $54(132)$ & $1.0(0.6-1.5)$ & & \\
\hline \multicolumn{5}{|l|}{ Ever taken to dental care } \\
\hline No & $51(159)$ & 1 & 1 & 1 \\
\hline Yes & $85(29)^{b}$ & $5.5(2.0-14.7)^{\mathrm{g}}$ & $3.1(1.1-8.9)$ & $3.1(1.1-8.9)$ \\
\hline \multicolumn{5}{|l|}{ Ever toothache } \\
\hline No & 48 (139) & 1 & 1 & 1 \\
\hline Yes & $89(49)^{b}$ & $8.9(3.7-21.3)^{9}$ & $7.8(2.9-20.6)$ & $7.8(3.0-20.7)$ \\
\hline \multicolumn{5}{|l|}{ Breastfeeding duration } \\
\hline $0-6$ months & $54(30)$ & 1 & & \\
\hline 7-12 months & $50(65)$ & $0.9(0.5-1.6)$ & & \\
\hline$>12$ months & $59(92)$ & $1.2(0.7-2.3)$ & & \\
\hline \multicolumn{5}{|c|}{ Mother /caretaker characteristics } \\
\hline \multicolumn{5}{|l|}{ Tooth brushing } \\
\hline No & $49(86)$ & 1 & 1 & 1 \\
\hline Yes & $60(99)^{\mathrm{a}}$ & $1.5(1.0-2.4)^{f}$ & $1.6(1.0-2.8)$ & $1.6(1.0-2.6)$ \\
\hline \multicolumn{5}{|l|}{ Caries experience } \\
\hline$D M F T=0$ & $44(37)$ & 1 & 1 & 1 \\
\hline$D M F T>0$ & $58(151)^{a}$ & $1.7(1.0-2.8)^{f}$ & $1.6(1.0-2.8)$ & $1.6(1.0-2.8)$ \\
\hline \multicolumn{5}{|l|}{ Marital status } \\
\hline Single/divorced, widow & $55(42)$ & 1 & & \\
\hline Married/cohabiting & $54(145)$ & $1.0(0.6-1.6)$ & & \\
\hline \multicolumn{5}{|l|}{ Type of income } \\
\hline No regular & $51(116)$ & 1 & 1 & 1 \\
\hline Regular & $62(72)^{a}$ & $1.6(1.0-2.5)^{f}$ & $1.7(1.0-2.8)$ & $1.7(1.0-2.8)$ \\
\hline \multicolumn{5}{|l|}{ Educational level } \\
\hline No- end of Primary school & $60(184)$ & 1 & & \\
\hline Middle/high school & $40(122)$ & $1.1(0.7-1.7)$ & & \\
\hline
\end{tabular}

Intra class correlation $\sim 0$

Dmft/DMFT Decayed, missing, filled teeth ${ }^{\mathrm{a}} p<0.05 ; \mathrm{X}^{2}$ test $^{\mathrm{b}} p<0.001 ; \chi^{2}$ test, ${ }^{\mathrm{c}}$ Ordinary least square, ${ }^{\mathrm{d}}$ Random intercept model, ${ }^{\mathrm{e}}$ Odds ratio $(95 \%$ confidence interval), ${ }^{\mathrm{f}} p<0.05,{ }^{\mathrm{g}} p<0.001$

oral health indicators of HEU and HUU children was available for comparison. The prevalence of untreated caries and quality of life impacts was high in this study population with 60 and $48 \%$ of respectively, HUU and HEU children showing untreated caries and 22\% HUU and 12\% HEU children reporting oral health related quality of life impacts. Compared with HUU community controls, the present study showed non-significant odds of caries experience and ECOHIS scores in HEU children who were breastfed under protection of periexposure prophylaxis for a postnatal period of 50 weeks. Nevertheless, independent of children's HIV-1 exposure 
Table 5 Child- and family oral health related quality of life scores (ECOHIS>0) by HIV-1 exposure status, socio-demographic -clinical and behavioral factors. Unadjusted and adjusted ordinary logistic regression and mixed model logistic regression

\begin{tabular}{|c|c|c|c|c|}
\hline Child characteristics & Unadjusted & Univariate OLR & Adjusted OLR & Adjusted RIM \\
\hline HIV-status & $\%(n)$ & OR $(95 \% \mathrm{Cl})^{\mathrm{e}}$ & OR $\left(95 \% \mathrm{Cl}^{\mathrm{e}}\right.$ & $\overline{\text { OR }(95 \% \mathrm{Cl})^{\mathrm{e}}}$ \\
\hline HIV unexposed- uninfected & $22(38)$ & 1 & 1 & 1 \\
\hline HIV exposed uninfected & $12(20)^{\mathrm{a}}$ & $0.5(0.3-0.9)^{\mathrm{a}}$ & $1.5(0.5-4.4)$ & $1.4(0.4-4.8)$ \\
\hline \multicolumn{5}{|l|}{ Sex } \\
\hline Male & $17(29)$ & 1 & & \\
\hline Female & $18(29)$ & $1.1(0.6-1.9)$ & & \\
\hline \multicolumn{5}{|l|}{ Age } \\
\hline 5 year & $13(13)$ & 1 & & \\
\hline 6-7 year & $19(46)$ & $1.6(0.8-3.1)$ & & \\
\hline \multicolumn{5}{|l|}{ Ever taken to dental care } \\
\hline No & $14(42)$ & 1 & 1 & 1 \\
\hline Yes & $48(16)^{b}$ & $5.9(2.8-12.6)^{f}$ & $4.2(1.9-9.3)$ & $5.0(1.8-13.6)$ \\
\hline \multicolumn{5}{|l|}{ Caries experience } \\
\hline $\mathrm{dmft}=0$ & $7(10)$ & 1 & 1 & 1 \\
\hline$d m f t>0$ & $26(48)^{b}$ & $5.2(2.5-10.7)^{f}$ & $4.3(2.0-9.1)$ & $4.6(2.0-10.6)$ \\
\hline \multicolumn{5}{|l|}{ Breastfeeding duration } \\
\hline $0-6$ months & $14(8)$ & 1 & 1 & 1 \\
\hline $7-12$ months & $11(14)$ & $0.7(0.3-1.9)$ & $0.7(0.3-2.0)$ & $0.8(0.3-2.3)$ \\
\hline$>12$ months & $23(36)^{a}$ & $1.8(0.8-4.3)^{\mathrm{g}}$ & $2.2(0.6-8.3)$ & $2.3(0.5-10.0)$ \\
\hline
\end{tabular}

Mother /caretaker characteristics

Tooth brushing

$\begin{array}{lll}\text { No } & 17(28) & 1 \\ \text { Yes } & 18(29) & 1.1(0.6-1.9) \\ \text { Caries experience } & & \\ \text { DMFT }=0 & 13(11) & 1 \\ \text { DMFT }>0 & 19(48) & 1.6(0.8-3.4)\end{array}$

Marital status

$\begin{array}{lll}\text { Single/divorced, widow } & 13(10) & 1 \\ \text { Married/cohabiting } & 19(48) & 1.5(0.7-3.0)\end{array}$

Type of income

$\begin{array}{lll}\text { No regular } & 15(33) & 1 \\ \text { Regular } & 21(24) & 1.6(0.9-2.8) \\ \text { Educational level } & & 1 \\ \text { Primary school } & 19(23) & 0.8(0.4-1.8) \\ \text { End of primary school } & 15(10) & 1.0(0.5-1.8) \\ \text { Middle/high school } & 19(23) & \end{array}$

Intra class correlation $(95 \% \mathrm{Cl}) ; 0.2(0.0-0.7)$

dmft/DMFT Decayed, missing, filled teeth ${ }^{\mathrm{a}} p<0.05 ; \mathrm{x}^{2}$ test, ${ }^{\mathrm{b}} \mathrm{p}<0.001 ; \mathrm{X}^{2}$ test, ${ }^{\mathrm{c}}$ Ordinary least square, ${ }^{\mathrm{d}}$ Random intercept model, ${ }^{\mathrm{e}}$ Odds ratio $(95 \%$ confidence interval), ${ }^{\mathrm{f}} p<0.001,{ }^{\mathrm{g}} p<0.05$

status, the odds of children's caries experience increased with caretakers' own caries experience and regular income status. Similarly, odds ratios of having quality of life impacts increased with child's caries experience and frequency of dental care visiting.
Strengths of this study include its nested long-term cohort design regarding the HEU children, and its use of a matched group of community HUU comparators. Previous studies have been criticized of using control groups of HUU children that differ significantly in 
socioeconomic status or breastfeeding patterns, thus leading to serious confounding $[19,20]$. Contrary to the situation in many high income countries where most HEU children come from marginalized sectors of the society, HIV-1 epidemic in low income countries is more generalized across the socioeconomic spectrum and thus HEU and HUU children are socio-economically more comparable [19]. Another strength is the strict characterization of the HIV-1 status of mothers and their children as well as use of a validated measure of ECOHIS scores. Statistical models for cluster adjustment are not well established in dental public health research $[36,37]$. Thus, the use of novel statistical methods in the present study stands as a methodological contribution to the scientific literature by accounting for the clustered structure in matched group data. The main limitation is the difference in follow-up between HEU and HUU children through lack of a concomitant control group. Missing data and loss to follow-up might also limit the interpretation of the findings as the present analyses might suffer from being underpowered. It is important to note that the caries prevalence in primary teeth of the HEU children might have been underestimated as only children passively followed up were included in the analyses and those who might have died before follow-up examination were not part of the group analyzed. Low statistical power due to the limited sample size available at follow-up may have been responsible for the absence of statistically significant association between HIV-1 exposure groups and dental caries as well as oral health related quality of life. Post-hoc power calculation was $61 \%$. There also could have been.

The present findings did not support the expectation that pre- and post-natal HIV-1exposure adversely affect dental caries in primary teeth nor oral health related quality of life of 5-7 years old children in Uganda. In this aspect, the present study accords with some previous African studies but contrasts with others investigating aspects of HEU children's general health and developmental delays [38-41]. The caries prevalence of 5-7-year-old HEU and HUU children observed in this study is comparable to that of children in the Ugandan general child population of similar age, previously estimated to range from 38 to $65 \%$ [31, 42]. Previous studies have reported on worse oral health among children with HIV-1 compared to healthy children of the same age group [11, 43]. Studies from low-income countries have reported on prevalence of caries in the deciduous teeth of children with HIV-1 ranging between 55 and 64\%, which is comparable to the prevalence reported in some studies of uninfected children in Uganda as well as among the community controls participating in the present study [9, 44]. Recently, a study of children with HIV-1 and HIV-exposed uninfected children /adolescents in the United States showed that participants with HIV-1 were more likely to present with caries in primary teeth, whereas caries experience in the permanent dentition and periodontal disease markers did not differ between the two groups [14]. Another study from Nigeria involving children with HIV, HUU and HEU showed that HEU children might not have a different caries experience than HUU children [41]. In accordance with the findings of this study, a Ugandan study revealed that quality of life impacts were similar for perinatally HEU and HUU community controls [27].

The prevalence of dental caries and oral impact scores were higher among HUU children than among their HEU counterparts. On the other side there were indications of a minor developmental delay in eruption of permanent teeth among HEU children presenting with significantly higher number of non-exfoliated primary teeth than HUU children [45]. The present findings both accord with and contradict previous studies from subSaharan Africa reporting on increased risk of developmental delays and poor nutritional status among HEU as compared to HUU children [20-22]. Notably, studies indicate that before the universal availability of antiretroviral therapy, ART, HEU children had higher mortality-, morbidity and growth failure risk than HUU children, whereas later studies from the ART era show heterogeneous findings [22]. In a meta-analytic review of neurodevelopment in young children born to mothers with HIV, Mc Henry et al. [40] found that children with HIV1 and HEU had worse health outcomes than their HUU counterparts and moreover, that HEU children without exposure to ART had worse health outcomes than their ART exposed counterparts. The HEU children in the present study were considered to be at low risk as they received infant prophylaxis (either lamivudine 3TC or boosted lopinavir/ritonavir, LPV/r daily) throughout the 50 weeks of breastfeeding in addition to standard prevention during delivery, complicating the natural history of HIV-1 exposure. In addition, the mothers with HIV-1 included were immune competent with $\mathrm{CD} 4$ counts > 350 cells $/ \mu \mathrm{L}$ when recruited into the cohort. Evidently, HEU children born to mothers with more severe disease assessed by the CD4 count present with higher rates of morbidity than those born to mothers with less sever disease $[19,20]$. Moreover, ART related improvements in the immune function of the HEU children participating in this study might have reduced their infection susceptibility generally and thus their vulnerability to caries in the deciduous dentition $[19,20]$. However, there might be other less measured extrinsic factors such as contact with the health system and years of counselling which may have altered help-seeking behavior and awareness around one's own and the child's health which may have influenced the behavior and ultimately 
the oral health. We have not controlled for all potential confounding factors.

Independent of children's HIV-1 exposure, and consistent with previous studies from sub Saharan Africa, caries in primary teeth and oral health related quality of life varied significantly according to child- and caretakers' clinical and behavioral characteristics $[4,8,31$, 33]. Thus, caretakers' caries experience was strongly associated with the caries experience of their offspring [8, 33]. Several plausible mechanisms are available for the relationship between caretakers and children's caries experience, such as mother to child transmission of genetic factors and shared oral habits between members of the whole family [8]. Finally, caries experience in HEU children was a strong covariate of their oral quality of life $[40,42]$. This finding indicates that ECOHIS has satisfactory criterion validity when utilized in the context of HEU and HUU Ugandan children [8, 33].

\section{Conclusion}

The prevalence of untreated caries in deciduous teeth and quality of life impacts was high in this study population. HIV-1 exposed uninfected children were not more likely than HUU children to experience dental caries or impaired oral health related quality of life. Given the global expansion of the HEU child population, the present findings indicating no adverse effect of pre- and postnatal HIV-1 exposure on caries in primary teeth are reassuring.

\section{Supplementary information}

Supplementary information accompanies this paper at https://doi.org/10. 1186/s12889-020-08564-1.

Additional file 1. Evaluation of effects of interaction of covariates with HIV-1 (EBF) exposure regressed with dental caries experience. Data indicating the individual association of the main exposure with dental caries experience as well as the effects of the main exposure and other covariates with dental caries experience.

\section{Abbreviations}

ARV: Antiretroviral; ART: Antiretroviral therapy; Cl: Confidence interval; CIS: Child impact scale; ECC: Early childhood caries; ECOHIS: Early childhood oral health impact scale; DMFT/ dmft: Decayed missing filled teeth surfaces; FIS: Family impact scale; HAART: Highly active antiretroviral therapy; HIV: Human deficiency virus; HEU: HIV exposed uninfected; HUU: HIV unexposed uninfected; ICC: Inter class correlation; IQR: Interquartile range; OR: Odds ratio; OLR: Ordinary logistic regression; PMTCT: Prevention of mother to child transmission; PROMISE-PEP: Promoting infant health and nutrition in Sub-Saharan Africa: Safety and efficacy of infant Peri-Exposure Prophylaxis; PROMISE-PEP-M\&S: Promoting infant health and nutrition in SubSaharan Africa: Safety and efficacy of infant Peri-Exposure Prophylaxis to prevent HIV-1 transmission by breastfeeding-Mechanisms \& Safety; RIM: Random intercept model; WHO: World Health Organisation

\section{Acknowledgements}

This study used data collected in the context of the ANRS 12174 clinical trial and 12341 study. We are grateful to the ANRS 12174 and 12341 for sharing data and giving us the opportunity to carry out oral assessments.

\section{Authors' contributions}

NB, AN, IMSE JKT, LTF and SAL made contributions to conception and design of the study. NB, AN and JKT contributed to data acquisition. NB, AN, IMSE, JKT, LTF and SAL analysed, interpreted, drafted and gave final approval to this version of the manuscript.

\section{Author information}

NB is a dentist and is a postdoctoral fellow at the Department of Clinical Dentistry, University of Bergen, Norway. Årstadveien, 195009.

\section{Funding}

This study used data collected in the context of the ANRS 12174 clinical trial and 12341 study funded by the French National Institute of Health and Medical Research - National Agency for Research on AIDS and Viral Hepatitis (Inserm-ANRS), the European Developing Countries Clinical Trials Partnership (EDCTP; grant number CT.2006.33020.004), the Research Council of Norway (GlobVac grant number 183600) and the Total Foundation. NB benefited from a postdoctoral research position funded by the Department of Clinical Dentistry, Faculty of Medicine, University of Bergen.

\section{Availability of data and materials}

The datasets used and/or analysed during the current study are available from the corresponding author on reasonable request.

\section{Ethics approval and consent to participate}

Ethical clearance was obtained from the School of Medicine Research and Ethics committee, Makerere University (SOMREC)-REC - 030, Uganda National Council of Science and Technology (UNCST)-HS 2373 and the Regional Committee for Health and Medical Research (REK) - 2017/760/REC sør-øst C from Norway.

\section{Consent for publication}

Not applicable.

\section{Competing interests}

The authors declare that they have no competing interests.

\section{Author details}

${ }^{1}$ Department of Clinical Dentistry, University of Bergen, P. O Box 7800 5020, Bergen, Norway. ${ }^{2}$ Bergen Addiction Research Group, Department of Addiction Medicine, Haukeland University Hospital, Bergen, Norway. ${ }^{3}$ Department of Global Health and Primary Health Care, University of Bergen, Bergen, Norway. ${ }^{4}$ Department of Paediatrics and Child Health, School of Medicine, College of Health Sciences, Makerere University, Kampala, Uganda.

Received: 17 October 2019 Accepted: 20 March 2020

Published online: 30 March 2020

\section{References}

1. Kassebaum NJ, Bernabé E, Dahiya M, Bhandari B, Murray CJL, Marcenes W. Global burden of untreated caries: a systematic review and Metaregression. J Dent Res. 2015:94:650-8.

2. Thitasomakul S, Thearmontree A, Piwat S, Chankanka O, Pithpornchaiyakul W, Teanpaisan R, Madyusoh S. A longitudinal study of early childhood caries in 9-to 18-month-old Thai infants. Community Dent Oral Epidemiol. 2006;34: 429-36.

3. Meyer F, Enax J. Early childhood caries: epidemiology, Aetiology, and prevention. Int J Dent. 2018;2018:1-7.

4. Masumo R, Bardsen A, Mashoto K, Astrom AN. Prevalence and sociobehavioral influence of early childhood caries, ECC, and feeding habits among 6-36 months old children in Uganda and Tanzania. BMC Oral Health. 2012:12:24.

5. Kumar VD. Early childhood caries--an insight. J Int Oral Health. 2010;2:1.

6. Congiu G, Campus G, Luglie PF. Early childhood caries (ECC) prevalence and background factors: a review. Oral Health Prev Dent. 2014;12:71-6.

7. Kim Seow W. Environmental, maternal, and child factors which contribute to early childhood caries: a unifying conceptual model. Int J Paediatr Dent. 2012;22:157-68.

8. Birungi N, Fadnes LT, Nankabirwa V, Tumwine JK, Astrom AN. Caretaker's caries experience and its association with early childhood caries and 
children's oral health-related quality of life: a prospective two-generation study. Acta Odontol Scand. 2016;74:605-12.

9. Rwenyonyi CM, Kutesa A, Muwazi L, Okullo I, Kasangaki A, Kekitinwa A. Oral manifestations in HIV/AIDS-infected children. Eur J Dent. 2011;5:291-8.

10. Nabbanja J, Gitta S, Peterson S, Rwenyonyi CM. Orofacial manifestations in HIV positive children attending Mildmay clinic in Uganda. Odontology. 2013;101:116-20.

11. de Aguiar RA, Portela MB, de Souza IP. The oral health of HIV-infected Brazilian children. Int J Paediatr Dent. 2013;23:359-65.

12. Meless D, Ba B, Faye M, Diby JS, N'Zore S, Datte S, Diecket L, N'Diaye C, Aka EA, Kouakou K, et al. Oral lesions among HIV-infected children on antiretroviral treatment in West Africa. Tropical Med Int Health. 2014;19:246-55.

13. Okunseri C, Badner V, Wiznia A, Rosenberg M. Prevalence of oral lesions and percent CD4+ T-lymphocytes in HIV-infected children on antiretroviral therapy. AIDS Patient Care STDs. 2003;17:5-11.

14. Moscicki AB, Yao TJ, Ryder MI, Russell JS, Dominy SS, Patel K, McKenna M, Van Dyke RB, Seage GR 3rd, Hazra R. The burden of Oral disease among perinatally HIV-infected and HIV-exposed uninfected youth. PLoS One. 2016; 11:e0156459.

15. Modi NS, Broyles NL, Montandon NM, Itoh NM, Ochanda NB, Langat NA, Sullivan ND, Dale NH. Beyond early infant diagnosis: changing the approach to HIV-exposed infants. J Acquir Immune Defic Syndr. 2018;78(Suppl 2): S107-14.

16. Progress report on the global plan towards the elimination of new HIV infections among children by 2015 and keeping their mothers alive. https:// www.unaids.org/sites/default/files/media_asset/JC2774_2015 ProgressReport_GlobalPlan_en.pdf. Acessed 25 Mar 2020.

17. Campion EW, Luzuriaga K, Mofenson LM. Challenges in the elimination of pediatric HIV-1 infection. N Engl J Med. 2016;374:761-70.

18. Evans $C$, Jones CE, Prendergast AJ. HIV-exposed, uninfected infants: new global challenges in the era of paediatric HIV elimination. Lancet Infect Dis. 2016:16:e92-e107.

19. Filteau S. The HIV-exposed, uninfected African child L'enfant africain exposé au VIH mais non infecté: une revue El VIH expuesto, el niño Africano no infectado: revisión. Tropical Med Int Health. 2009;14:276-87.

20. Evans C, Humphrey JH, Ntozini R, Prendergast AJ. HIV-exposed uninfected infants in Zimbabwe: insights into health outcomes in the pre-antiretroviral therapy era. Front Immunol. 2016;7:190.

21. Muhangi L, Lule SA, Mpairwe H, Ndibazza J, Kizza M, Nampijja M, Nakazibwe E, Kihembo M, Elliott AM, Webb EL. Maternal HIV infection and other factors associated with growth outcomes of HIV-uninfected infants in Entebbe, Uganda. Public Health Nutr. 2013;16:1548-57.

22. Rupérez M, González R, Maculuve SA, Quintó L, López Varela E, Augusto O, Vala A, Nhacolo A, Sevene EJP, Naniche D, et al. Maternal HIV infection is an important health determinant in non-HIV-infected infants. AIDS. 2017;31: $1545-53$.

23. Le Roux MS, Donald AK, Brittain KK, Phillips KT, Zerbe JA, Nguyen JK, Strandvik JA, Kroon JM, Abrams JE, Myer JL. Neurodevelopment of breastfed HIV-exposed uninfected and HIV-unexposed children in South Africa. AIDS. 2018;32:1781-91.

24. Powis MK, Slogrove LA, Mofenson LL. Protecting the health of our AIDS-free generation: beyond prevention of mother-to-child HIV transmission. AIDS. 2017:31:315-6.

25. Mebrahtu H, Simms V, Chingono R, Mupambireyi Z, Weiss HA, Ndlovu P, Malaba R, Cowan FM, Sherr L. Postpartum maternal mental health is associated with cognitive development of HIV-exposed infants in Zimbabwe: a cross-sectional study. AIDS Care. 2018;30(Suppl 2):74-82.

26. Lee GM, Gortmaker SL, McIntosh K, Hughes MD, Oleske JM. Quality of life for children and adolescents: impact of HIV infection and antiretroviral treatment. Pediatrics. 2006;117:273-83.

27. Nkwata AK, Zalwango SK, Kizza FN, Sekandi JN, Mutanga J, Zhang M, Musoke PM, Ezeamama AE. Quality of life among perinatally HIV-affected and HIV-unaffected school-aged and adolescent Ugandan children: a multidimensional assessment of wellbeing in the post-HAART era. Qual Life Res. 2017;26:2397-408.

28. Zalwango SK, Kizza FN, Nkwata AK, Sekandi JN, Kakaire R, Kiwanuka N, Whalen CC, Ezeamama AE. Psychosocial adjustment in perinatally human immunodeficiency virus infected or exposed children - a retrospective cohort study. J Int AIDS Soc. 2016;19:20694.

29. Webster KD, de Bruyn MM, Zalwango SK, Sikorskii A, Barkin JL, FamiliarLopez I, Musoke P, Giordani B, Boivin MJ, Ezeamama AE. Caregiver socioemotional health as a determinant of child well-being in school-aged and adolescent Ugandan children with and without perinatal HIV exposure. Tropical Med Int Health. 2019;24:608-19.

30. Nagot N, Kankasa C, Tumwine JK, Meda N, Hofmeyr GJ, Vallo R, Mwiya M, Kwagala M, Traore H, Sunday A, et al. Extended pre-exposure prophylaxis with lopinavir-ritonavir versus lamivudine to prevent HIV-1 transmission through breastfeeding up to 50 weeks in infants in Africa (ANRS 12174): a randomised controlled trial. Lancet. 2016;387:566-73.

31. Birungi N, Fadnes LT, Okullo I, Kasangaki A, Nankabirwa V, Ndeezi G, Tumwine JK, Tylleskar T, Lie SA, Astrom AN. Effect of breastfeeding promotion on early childhood caries and breastfeeding duration among 5 year old children in eastern Uganda: a cluster randomized trial. PLoS One. 2015;10:e0125352.

32. Pahel BT, Rozier RG, Slade GD. Parental perceptions of children's oral health: the early childhood Oral health impact scale (ECOHIS). Health Qual Life Outcomes. 2007;5:6.

33. Masumo R, Bardsen A, Mashoto K, Astrom AN. Child- and family impacts of infants' oral conditions in Tanzania and Uganda-- a cross sectional study. BMC Oral Health. 2012:5:538.

34. Who. Oral Health Surveys : Basic Methods. 5th ed. Albany: World Health Organization; 2013.

35. Galiwango RM, Musoke R, Lubyayi L, Ssekubugu R, Kalibbala S, Ssekweyama V, Mirembe V, Nakigozi G, Reynolds SJ, Serwadda D, et al. Evaluation of current rapid HIV test algorithms in Rakai, Uganda. J Virol Methods. 2013; 192:25-7.

36. Fleming PS, Koletsi D, Polychronopoulou A, Eliades T, Pandis N. Are clustering effects accounted for in statistical analysis in leading dental specialty journals? J Dent. 2013;41:265-70.

37. Gülcan F, Ekbäck G, Ordell S, Lie SA, Åstrøm AN. Social predictors of less frequent dental attendance over time among older people: populationaveraged and person-specific estimates. Community Dent Oral Epidemiol. 2016:44:263-73.

38. Springer PE, Slogrove AL, Laughton B, Bettinger JA, Saunders HH, Molteno CD, Kruger M. Neurodevelopmental outcome of HIV-exposed but uninfected infants in the mother and infants health study, Cape Town, South Africa. Tropical Med Int Health. 2018;23:69-78.

39. Chaudhury S, Williams PL, Mayondi GK, Leidner J, Holding P, Tepper V Nichols S, Magetse J, Sakoi M, Moabi K, et al. Neurodevelopment of HIVexposed and HIV-unexposed uninfected children at 24 months. Pediatrics. 2017;140:4.

40. McHenry MS, McAteer $\mathrm{Cl}$, Oyungu E, McDonald BC, Bosma CB, Mpofu PB, Deathe AR, Vreeman RC. Neurodevelopment in young children born to HIVinfected mothers: a meta-analysis. Pediatrics. 2018;141:e20172888.

41. Coker M, El-Kamary S, Enwonwu C, Blattner W, Langenberg P, Mongodin E, Akhigbe P, Obuekwe O, Omoigberale A, Charurat M. Perinatal HIV infection and exposure and their association with dental caries in Nigerian children. Pediatr Infect Dis J. 2017;37:59-65.

42. Kiwanuka SN, Åstrøm AN, Trovik TA. Dental caries experience and its relationship to social and behavioural factors among 3-5-year-old children in Uganda. Int J Paediatr Dent. 2004;14:336-46.

43. Cerqueira DF, Portela MB, Pomarico L, de Araujo Soares RM, de Souza IP, Castro GF. Oral Candida colonization and its relation with predisposing factors in HIV-infected children and their uninfected siblings in Brazil: the era of highly active antiretroviral therapy. J Oral Pathol Med. 2010;39:188-94.

44. Masiga MA, M'Imunya JM. Prevalence of dental caries and its impact on quality of life (QoL) among HIV-infected children in Kenya. J Clin Pediatr Dent. 2013;38:83-7.

45. Bastos JL, Peres MA, Peres KG, Barros AJD. Infant growth, development and tooth emergence patterns: a longitudinal study from birth to 6 years of age. Arch Oral Biol. 2007;52:598-606.

\section{Publisher's Note}

Springer Nature remains neutral with regard to jurisdictional claims in published maps and institutional affiliations. 